\title{
PNEUMATIC LINEAR INCREMENTAL ACTUATOR
}

\author{
Constantin Bucșan ${ }^{1}$, Mihai Avram² \\ 1,2 POLITEHNICA University of Bucharest \\ Spl. Independentei 313, Bucharest, Romania \\ constantinbucsan@yahoo.com, mavram02@yahoo.com
}

\begin{abstract}
The paper refers to a linear pneumatic actuator with incremental functioning developed by the authors. The structure of the actuator is presented and the theoretical aspects that are governing the actuator working are treated. On the basis of the functional model built by the authors, the static characteristic of the actuator is determined, theoretically and then experimentally. Finally, the solution found in order to optimize the performances of the actuator are presented.
\end{abstract}

Keywords: Linear Actuator, Pneumatic, Incremental, Positioning Accuracy.

\section{Introduction}

In controlling processes, pneumatic actuating systems are often used for positioning the actuated loads with an imposed accuracy. But increasing the positioning accuracy and especially maintaining the position are still unsolved problems. In addition, some perturbations (variations of the actuated load, variations of the supply pressure, flow loses etc.) occur while moving to the target position and also while the load stands in that position, perturbations that are difficult to be controlled and that influence the dynamics of the movement and the maintaining of the position. It is known that stopping the mobile assembly of a pneumatic motor (including the actuated load) can be performed with accuracy at the stroke ends or using mechanical stops placed along the working stroke. If such stops are not used, stopping in any other position of the working stroke is difficult to be controlled due to the compressibility of the air. However it is possible to develop pneumatic motors that allow stopping with accuracy in a limited number of positions.

Pneumatic step by step motors are of large interest. With these motors the effector (rod or shaft) can be positioned in a finite number of points along the working stroke. For every command signal the effector moves with one step. In the case of proper functioning, the number of effectuated steps is equal with the number of the command signals, and so an accurate positioning is achieved without using a position transducer, even if the actuated load varies.

There are many types of pneumatic step by step motors, but the most used are those with cam mechanism and with harmonic gear $[1,2,3]$.

The step by step pneumatic rotational motors with harmonic gear are characterized by high ratio and small dimensions, they are simple and very compact, their shafts are coaxial and their positioning accuracy is good. The harmonic gears transmit the movement with low backlash or even without backlash in the case of the gears with flexible cylindrical gear wheel. Another characteristic of this type of motors is the very small angular step and the high motor torque.

From the structure point of view the harmonic gears are of two main types: with rigid conical gear wheels and with cylindrical gear wheels with internal engagement, one of which is a flexible gear wheel. Both types were used to build pneumatic step by step motors: pneumatic step by step motors with harmonic gear with conical gear wheels [1,4], pneumatic step by step motors with harmonic gear with cylindrical gear wheels with internal engagement [6], or pneumatic step by step motors produced by BIBUS [5].

This paper presents a linear pneumatic actuator with incremental functioning developed by the authors in order to achieve accurate positioning in a finite number of points along the working stroke.

\section{The Arrangement of The Proposed Actuator}

The working principle of the proposed actuator is that of a cam mechanism, but with the cam being actuated by the follower.

A classic cam mechanism transforms the movement of the cam into an alternative translation movement of the follower according to the cam profile. Inverting the functions, the follower becomes the leading element and the cam the led element. 
Figure 1 shows the working principle of such a motor [3].

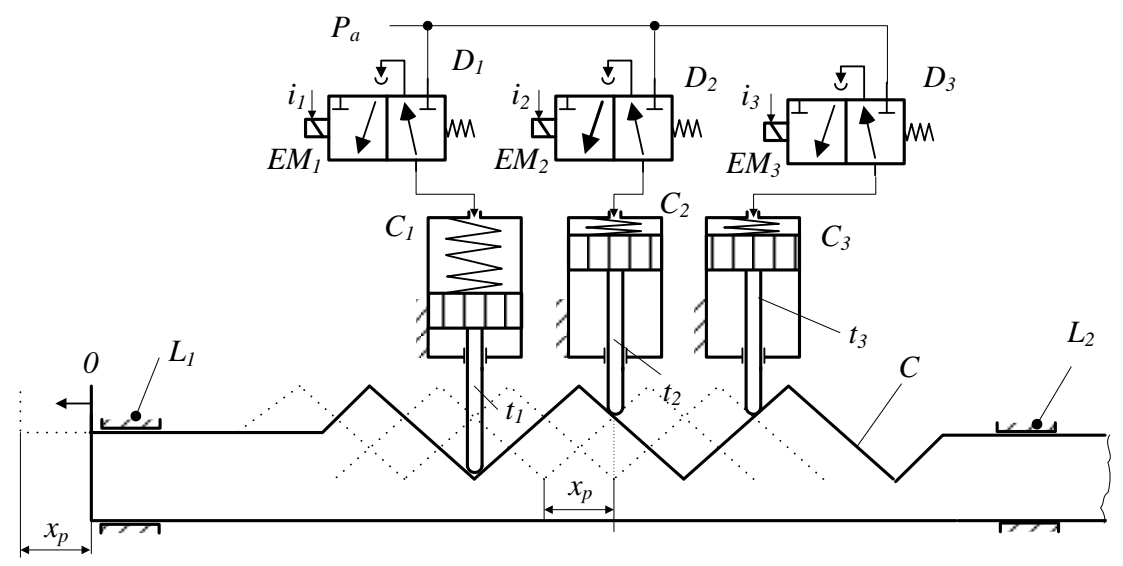

Fig. 1. The principle scheme of the proposed linear actuator

In order to obtain an incremental movement, the profile of the cam $C$ is composed of successive identic up and down segments, so the cam appears to be a rack. There are used three followers instead of one, placed as shown in the figure. The followers $t_{1}, t_{2}$ and $t_{3}$ are the rods of three pneumatic cylinders $C_{1}, C_{2}$ and $C_{3}$. Always one of the followers is indexed, as it is $t_{1}$ in the figure. That means at that moment the valve $D_{1}$ corresponding to the cylinder $C_{1}$ is commanded. In order to obtain the movement with one step to the left, a command signal $i_{2}$ is applied to the valve $D_{2}$ corresponding to the cylinder $C_{2}$ and the previous

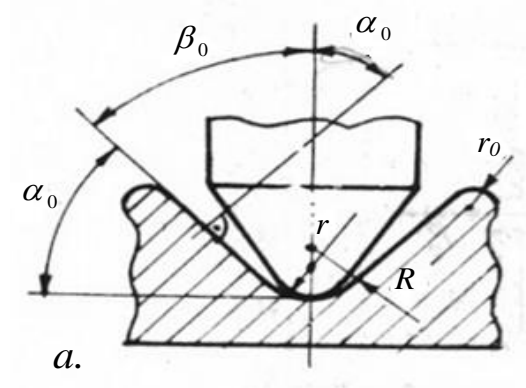

command $i_{1}$ is cancelled. So the movement to the left is obtained commanding the cylinders with the sequence $i_{1}-i_{2}-i_{3}-i_{1}-\ldots$. of signals. The movement in the opposite direction is obtained commanding the cylinders with the sequence $i_{1}-i_{3}-i_{2}-i_{1}-\ldots .$. of signals. Usually the follower has a spherical or conical shape at the zone in contact with the cam. The profile of the cam can be of two types, as shown in Figure 2.

b.

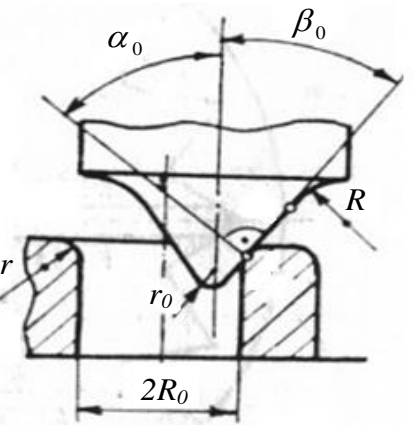

Fig. 2. Variants of cam profile

Figure 2,a shows a cam with triangular teeth with rounded tips, while Figure 2,b the profile of the tooth is shaped on the follower end, which is a cone with rounded tip, and the cam has cylindrical holes with a connection radius $r$. The second variant is more used due to the simplest necessary machining technology.

\section{The Construction of the Actuator}

Figure 3 shows a 3D view of the proposed actuator. The slider $\mathrm{S}$, guided on reels, has a number of calibrated holes machined perpendicular to the longitudinal axis and equidistant [3]. At the right end of the slider a hydraulic damper D is mounted.

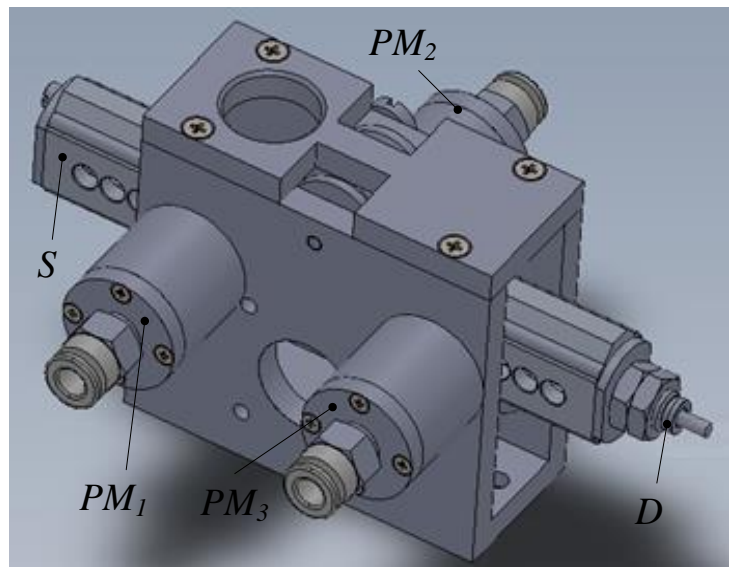

Fig. 3. A 3D view of the functional model of the actuator 
The 3 followers are machined on the rods of the pneumatic linear motors $P M_{1}, P M_{2}$ și $P M_{3}$. These motors are mounted on the actuator body in precise positions [2]. At any time one of the followers is indexed (its axis coincides with the axis of a hole), while the other two followers are offset with one step in one direction or another from the axis of the adjacent holes.

\section{The Theoretical Determining of the Static Characterisitc of the Actuator}

In order to use such a linear motor is important to know its static characteristic, represented by the relation between the follower movement and the obtained force generated on the moving direction of the slider, for certain supply pressures.

Figure 4 shows the engagement zone between the follower and the hole of the slider. In order to determine the equation of the slider movement $y$ depending on the follower movement $\mathrm{x}$, trigonometric relations in the triangles $O A B$ and $O A O_{1}$ are used:

$$
\begin{aligned}
& y=y_{0}+\delta=y_{0}+x \tan \alpha \\
& y_{0}=\frac{d_{D}}{2}+\frac{D}{2}-y_{p}+a \\
& a=r(1-\cos \alpha)
\end{aligned}
$$

and we get:

$$
y=\frac{d_{D}}{2}+\frac{D}{2}-y_{p}+r(1-\cos \alpha)
$$

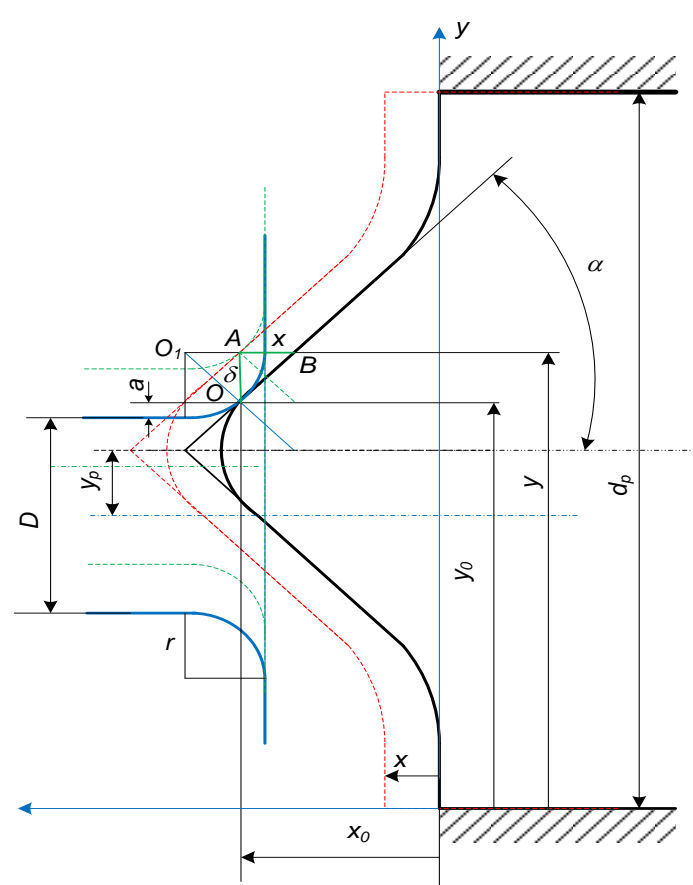

Fig. 4. The movement calculus scheme
Replacing with the numerical values corresponding to the experimental model: $\alpha=45^{\circ}$, $d_{p}=16 \mathrm{~mm}, \quad D=5.2 \mathrm{~mm}, \quad r=1.6 \mathrm{~mm}$ si $y_{p}=2 \mathrm{~mm}$ the following relation is obtained:

$$
y=9.0688+x
$$

In order to determine the actuating force of the slider, the equilibrium equation is written, using the notations in Figure 5:

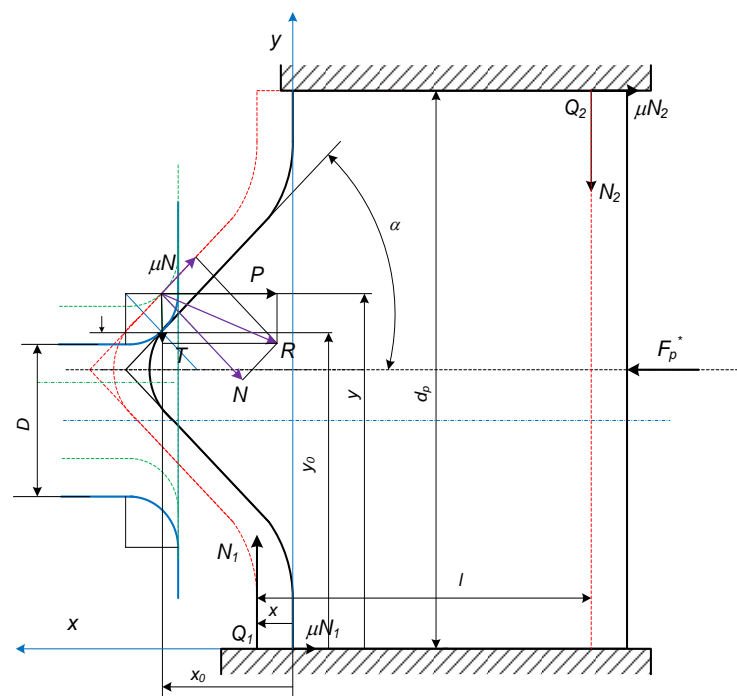

Fig. 5. The forces calculus scheme

$$
\begin{aligned}
& \left\{\begin{array}{c}
\left(\sum M\right)_{Q_{1}}=0 \\
\left(\sum M\right)_{Q_{2}}=0 \\
\sum F_{1}=0
\end{array}\right. \\
& T=R \cos \left(\alpha+\varphi_{0}\right), \\
& P=R \sin \left(\alpha+\varphi_{0}\right), \\
& F_{p}^{*}=F_{p}+F_{\alpha}-F_{f}
\end{aligned}
$$$$
\left\{\begin{array}{c}
T\left(x_{0}-x\right)-P y-N_{2} l-\mu N_{2} d_{p}+F_{p}^{*} \frac{d_{p}}{2}=0 \\
T\left(l+x_{0}-x\right)+P\left(d_{p}-y\right)-N_{1} l+\mu N_{1} d_{p}-F_{p}^{*} \frac{d_{p}}{2}=0 \\
P+\mu N_{1}+\mu N_{2}-F_{p}^{*}=0
\end{array}\right.
$$

where $F_{a}$ is the force of the spring used to overcome the friction force $F_{f}$, maintaining the permanent contact between the follower and the slider.

Considering $F_{a} \approx F_{f}$, we get:

$$
F_{p}^{*} \approx F_{p}=\frac{\pi d_{p}^{2}}{4} p_{a}
$$

Solving the equation system (7) we get: 


$$
R=\frac{\frac{\mu d_{\eta}^{2}}{4} \mathrm{p}_{a}}{\sin \left(\alpha+\varphi_{0}\right)+\frac{H}{l}\left[\left(l+2 x_{0}-2 x\right) \cos \left(\alpha+\varphi_{0}\right)+\left(d_{p}-2 y\right) \sin \left(\alpha+\varphi_{0}\right)\right]}
$$

The force generated on the slider movement direction is:

$$
T=R \cos \left(\alpha+\varphi_{0}\right)
$$

Which represents the equation of the static characteristic of the linear actuator.

Replacing with the numerical values corresponding to the experimental model:

$$
\begin{aligned}
& \alpha=45^{\circ}, d_{p}=16 \mathrm{~mm}, \mu=0.15, \\
& \varphi_{0}=\tan ^{-1} \mu=8.5^{\circ} \text { ssi } l=20 \mathrm{~mm}, \text { we get: } \\
& T=\frac{47.8124}{0.9715-0.02098 x}
\end{aligned}
$$

Figure 6 shows the static characteristic of the linear incremental pneumatic actuator.

The effective stroke of the follower is between 0 and $x_{\max }=2 \mathrm{~mm}$.

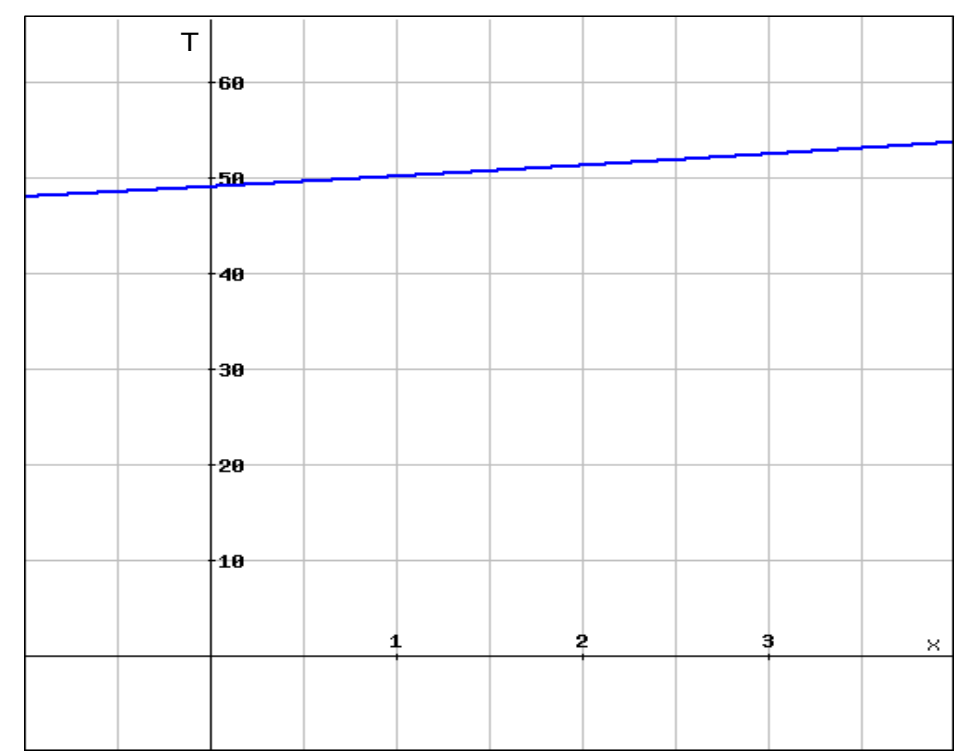

Fig. 6. The static characteristic of the linear incremental pneumatic actuator

\section{The Experimental Determining of the Static Characteristic of the Actuator}

Figure 7 shows the principle scheme of the stand used to determine experimentally the static characteristic of the linear actuator.

The experimental model of the linear incremental pneumatic actuator 1 is actuated using the controlled valves 2,3 and 4 , supplied by the pressure regulator 5 and commanded by the microcontroller 11. A force transducer 6 in mounted on the end of the actuator slider, which is pushed against the rod of a hydraulic cylinder 7 , that has a choke 8 mounted between the orifices of its two chambers.

The microcontroller commands, according to the algorithm implemented in the working program, the three distributors with the signals $u_{1}, u_{2}$ and $u_{3}$, so as the slider moves and pushes the rod of the hydraulic cylinder. The choke 8 is used to vary the resistant force at the rod of the hydraulic cylinder.

The microcontroller receives the signal $u_{p}$ (proportional with the adjusted pressure $p$ ) from the pressure transducer 9, the signal $u_{y}$ (proportional with the movement $y$ of the slider) from the displacement transducer 10 , and also the signal $u_{f}$ (proportional with the force $F$ developed by the actuator) from the force transducer 6. So, the maximum force developed by the actuator for every adjusted pressure $p$ can be determined. 


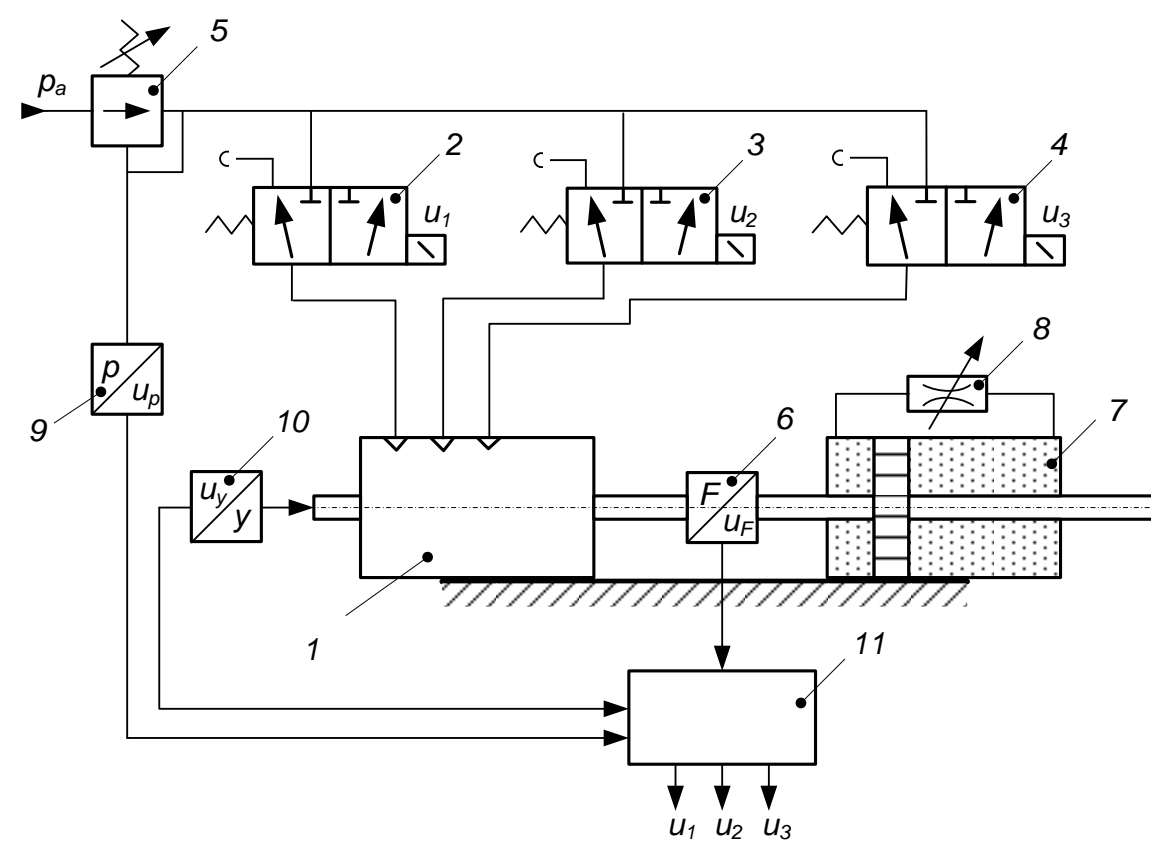

Fig. 7. The principle scheme of the experimental stand

The experimental results confirmed the theoretical approach and they indicated some directions to follow when designing the prototype of the linear incremental pneumatic actuator in order to optimize its behavior. Some of these directions are technological (materials, hardness and roughness of the surfaces in contact etc.) and the other ones are related to the algorithms implemented in the command program.

\section{References}

[1] Demian, T. and Banu, V.: Micromotoare Pneumatice liniare şi Rotative, Editura Tehnică, Bucureşti (1984).
[2] Banu, V.: Cu privire la principiile de realizare a motoarelor pneumatice pas cu pas, Construcţia de maşini, nr.5 (1979).

[3] Banu, V., Avram, M.: Construction and Operation of an Incremental Pneumatically Actuated Linear Unit", Mecatronica, nr.2 (2008).

[4] Baliklock, P.M.: Development of Pneumatic Stepping Motor, IFAC Symposium on Fluidics, London (1968).

[5] https://www.bibus.uk/productssolutions/pneumatics/air-motors/.

[6] Roberts, E.W.: Space tribology: its role in spacecraft mechanisms, Journal of Physics D: Applied Physics, vol.45, no. 50 (2012).

[7] Avram, M., Bucșan, C.: Sisteme de acționare pneumatice inteligente, Editura Politehnica PRESS, Bucuresti (2014). 